\title{
XXVIII. On the fundamental equations of electrodynamics and Crémieu's experiment
}

\author{
H.C. Pocklington M.A. D.Sc.
}

To cite this article: H.C. Pocklington M.A. D.Sc. (1901) XXVIII. On the fundamental equations of electrodynamics and Crémieu's experiment , Philosophical Magazine Series 6, 1:3, 325-330, DOI: $10.1080 / 14786440109462617$

To link to this article: http://dx.doi.org/10.1080/14786440109462617

册 Published online: 16 Apr 2009.

Submit your article to this journal $[\pi$

ЏII Article views: 2

Q View related articles $\square$ 
XXVIII. On the Fundamental Equations of Electrodynamics and Crémieu's Experiment. By H. C. Pocklington, M.A., D.Sc.*

1. THE object of this paper is to deduce the equations of 1 the electronagnetic field from the results of experiment combined with the simplest possible assumptions, and to use them to discuss the question whether a moving charge produces a magnetic field, and whether the sudden charging of a rotating disk will cause an induced current in a coil surrounding it (Crémieu's experiment). The results of the investigation are that Hertz's form of Maxwell's equations can be obtained with the aid of assumptions so simple as to be almost beyond doubt, and with them the theorem that a moving charge produces a magnetic field, but that it is somewhat doubtful whether an induced current should theoretically be observed in Crémieu's experiment. The assumption that the phenomena are mechanical and therefore satisfy certain reciprocal relations is not made, and no assumptions are made as to the equations that are to be satisfied at the surface of a moving body or in its substance. We use Quaternions on account of the difficulty of expressing in Cartesians some of the ideas involved.

2. We shall consider the electric force first.

1. EXPERIMENTAL FACT. The other is homogeneous and isotropic.-This follows from the independence of the phenomena of light, electrostatics and current electricity on any direction or position fixed in space.

II. Assumption. The electric force satisfies differential equations. - The analogous proposition is true for the case of the motion of an elastic solid or of a membrane. It is not true for the motion of the surface of a liquid oscillating under gravity.

III. Expertmential Fact. The differential equations are linear.-For otherwise two trains of waves of light could not be propagated through the same space independently of each other.

IV. ExPRRIMnNTAI FACT. The velocity of propagation of all waves is the same.-This is shown by experiments on the velocity of light of different wave-lengths.

V. Experimental Fact. Waves are propagated through the ather without suffering absorption.-Otherwise the ratio of the magnitudes of two stars, one near the pole of the ecliptic, the other near the ecliptic, would depend on the time of year when the observation was made.

* Communicated by the Author. 
326 Dr. Pocklington on the Fundamental Equations of

VI. Experimental Fact. When the electric force is independent of the time its convergence is zero. -For in all that follows we only consider points in the free æother, and assume that the xther itself cannot be electrified.

3 . Let the electric force at any point be $\sigma$, where $\sigma$ is a function of the time $t$ and of the vector-coordinate $\rho$ of the point. By II. $\sigma$ satisfies differential equations, which, since $d i d x=-\bar{S} i \nabla$, ean be expressed in terms of $d / d t$ and $\nabla$. The resulting equations can by $I$. contain no constant vector, and by III. and I. they are linear and with constant scalar coefficients. If such an equation is scalar, it can only be of the form

$$
f\left(\frac{d}{d t}, \nabla\right) \mathrm{S} \nabla \sigma=0
$$

and thus $\sigma$ must satisfy either

$$
\mathrm{S} . \nabla \sigma=0 \text { or } f\left(\frac{d}{d t}, \nabla\right) \sigma=0 .
$$

If the latter equation is scalar, the same remark applies: and hence, finally, $\sigma$ satisfies either $\mathrm{S} . \nabla \sigma=0$ or a vector differential equation. In the latter case the equation satisfied cannot reduce to a sealar equation when $\sigma$ is independent of t. Hence, by Vl., $\sigma$ satisfies* the equation $S . \nabla \sigma=0$.

4. An equation satisfied by $\sigma$ may be vector. Using the formula $S \cdot \nabla \equiv 0$ when applied to $\sigma$ and its derivatives, we get $V \cdot \nabla \equiv \nabla$, and by these we can reduce any function of $\nabla$ to one containing only powers of $\nabla$. To discuss the form of this equation we must consider the plane wave. Let

$$
\sigma=\alpha e^{(p t+q \mathrm{~S} \beta \rho)} \overrightarrow{\sqrt{-1}},
$$

where $\beta$ is a unit vector and $p$ and $q$ are scalars. Then $d / d t \equiv p^{\sqrt{ }-1}, \nabla \equiv-q \sqrt{ }-1$. Substituting in the equations we get $\mathrm{S} \alpha \beta=0$ from that found in $\S 3$, and from our present equation we get one equation in $p$ and $q$ if only odd or only even powers of $\nabla$ enter ; or, in the other case, two such equations. This equation (or equations) can contain only even powers of $p$, as otherwise an exponential would enter into the final formula, thus contradicting $V$. The equation, which is therefore an equation in $p^{2}$, must give only positive values for $p^{2}$, for the same reason. It can only give one value, otherwise there would be more than one velocity for

* It may be objected that in the case of the motion of a compressible fluid, an equation of steady motion is $S \cdot \nabla \sigma=0$, where $\sigma$ is the vector velocity of the fluid at any point, whilst this equation does not hold if the motion is not steady. Here, however, the motion is not given by linear equations. 
Electrodynamics and Crémieu's Experiment.

the wave, which is in contradiction to IV. The equation is therefore

$$
p^{2} \mathrm{~F}(q)+f(q)=0 .
$$

The velocity of the wave is $v=p / q$, and by IV. is constant. This requires $f(q)=-v^{2} q^{2} \mathrm{~F}(q)$, and $\mathrm{F}(q)$ can be cancelled ont. Since only powers of $\nabla$ enter into the differential eqnation, the same must be true of it, and the equation can be reduced to

$$
\left(\frac{d^{2}}{d t^{2}}+v^{2} \nabla^{2}\right) \sigma=0 \text {. . . . . . . }
$$

5. We must now consider the magnetic fcrce.

VII. Experimental FAcT. The magnetic force in a plane wave is perpendicular to the electric force, and the ratio of the amplitudes of the two is independent of the wave-length.

VIII. Assumption. The magnetic force is connected with the electric force by a differential equation.

IX. Assumption. The component in any direction of the electric force produced by a changing magnetic field is equal to the induced electromotive force in an element of a wire lying in that direction.

$\mathrm{X}$. Defrinition. The unit electromotive force is that which is produced in a circuit by unit rate of change in the surfaceintegral of the magnetic force. - This definition implies that the electromotive force depends only on the rate of change of the surface-integral. This is proved below; the definition of the unit is all that is required from $X$.

6. Let the magnetic force be $\tau$. Then since $S . \nabla \equiv 0$ and $\mathrm{V} \cdot \nabla \equiv \nabla$, the differential equation can contain only powers of $\nabla$, and its solution will be

$$
\tau=\frac{f\left(\frac{d}{d t}, \nabla\right)}{\mathrm{F}\left(\frac{d}{d t}, \nabla\right)} \sigma .
$$

By using $v^{2} \nabla^{2} \equiv-d^{2} / d t^{2}$ this can be reduced to the form

$$
\tau=\left\{f_{1}\left(\frac{d}{d t}\right)+f_{2}\left(\frac{d}{d t}\right) \nabla\right\} \sigma,
$$

where $f_{1}, f_{2}$ may be of fractional form. From VII. we see that $f_{1}=$ const., $f_{2}=$ const. $/(d / d t)$, and hence

$$
\boldsymbol{\tau}=\left(e+\frac{g \nabla}{d^{\prime} d \bar{t}}\right) \sigma_{\bullet}
$$


328 Dr. Pocklington on the Fundamental Equations of

Taking now the case of plane waves, from VII. S $\tau \sigma=0$, and hence $e=0$, giving

$$
\frac{d \tau}{d t}=g \nabla \sigma .
$$

From this, the surface-integral of $d \tau / d t$ equals the line-integral of $g \sigma$. From IX. and X. we see that $g$ must be \pm 1 . We must take the negative sign if we chose the axes as usual.

Finally,

$$
\frac{d \tau}{d t}=-\nabla \sigma, \quad . \quad . \quad . \quad \cdot .
$$

which gives $\mathrm{S} \cdot \nabla \tau=0$.

7. From (1) and (2) we get

$$
\begin{aligned}
& \frac{d^{2} \sigma}{d t^{2}}=v^{2} \nabla \frac{d \tau}{d t} \\
& \frac{d \sigma}{d t}=v^{2} \nabla \tau+\text { const., }
\end{aligned}
$$

and the constant is clearly zero.

Finally, then, our assumptions lead to the equations of the field in Hertz's form,

$$
\begin{aligned}
\mathrm{S} \nabla \sigma & =0, \quad \mathrm{~S} \nabla \tau \\
\frac{d \sigma}{d t}=v^{2} \nabla \tau, & \frac{d \tau}{d t}=-\nabla \sigma . \quad . \quad . .
\end{aligned}
$$

8. We must now consider the case of a moving charge.

XI. Assumption. The motion of a body produces no motion in the oether through which it moves.-This has been verified experimentally for the case where the moving body rotates and always occupies the same space.

Let the charged body move with a constant velocity $\gamma$. Then $d / d t=\mathrm{S} \gamma \nabla()$, and from (3)

$$
\begin{aligned}
\mathrm{V} \cdot \nabla \tau & =\frac{1}{v^{2}} \frac{d \sigma}{d t} \\
& =\frac{1}{v^{2}}(\mathrm{~S} \gamma \nabla \cdot \sigma-\gamma \mathrm{S} \cdot \nabla \sigma)
\end{aligned}
$$

since $S \cdot \nabla \sigma=0$,

$$
=\frac{1}{v^{2}} \mathrm{~V} \cdot \nabla \mathrm{V} \gamma \sigma
$$

whence

$$
\tau=\frac{1}{v^{2}} \mathrm{~V} \boldsymbol{\gamma} \boldsymbol{}+\tau_{0}, \ldots . . \quad . \quad .
$$

where $\tau_{0}$ is any solution of $\mathrm{V} \cdot \nabla r_{0}=0, \quad \mathrm{~S} . \nabla \tau_{0}=0$. 
Hence $\tau$ is only derivable from a potential function at places where $\sigma$ vanishes.

In this equation $\sigma$ is the actual electric force. If the velocity is small compared with $v$, we may for an approximation put $\sigma$ equal to the electric force in a state of rest.

From the equations satisfied by $\tau_{0}$ the value of the lineintegral of $\tau_{0}$ is the same for all circuits that can be deformed into one another without leaving the free æother, in which alone we can be certain that the equations are satistied. If the charged body is not infinite in length, the circuit can be reduced to zero, and hence the line-integral is zero. The lineintegral of $\mathrm{V}_{\gamma \sigma}$ does not vanish, and thus the second term cannot cancel the first. Hence there must be some magnetic force around the moving body.

If the assumption XI. is not true, the velocity of the æther must, at a sufficient distance from the body, vary inversely as the cube of the distance. The magnetic force just found varies inversely as the square, and hence cannot be cancelled out by any effect that a motion of the æther can produce. Whether XI. be true or not, there must be some magnetic force produced.

If the body is infinitely long, the argument fails. If, however, the infinitely long body be cut through at any point, however narrow the gap may be, the argument applies.

$\boldsymbol{y}$. If the body moves inside an infinite tube the results of the last paragraph hold for the space inside the tube. In the case of the space outside there is some difficulty, as we cannot prove that the term $\tau_{0}$ is unimportant for our purpose. If, however, we take the tube to be of finite though considerable length, and suppose the body to be at a distance from the end, the argument applies; and the magnetic force outside, which reduces to $\tau_{0}$, has its line-integral of value zero. Hence, if everything is symmetrical about the axis of the tube, $\tau_{0}$ vanishes; and in this case at least there is no magnetic force outside the tube.

10. If we suppose the tube not to be continuous, but to consist, for example, of a gilt glass tube where the gilding is separated into a number of narrow rings by transverse scratches, it seems clear that, if unelectrified, it will produce little or no effect on the field of electric force in the case where a single charged body moves along the tube. There will therefore be an external magnetic field. If a number of such charged bodies separated by narrow gaps move along the tube, there must be an external magnetic field. An electrification of the tube sufficient to produce an electric field equal but opposite to the mean of the nearly constant

Phil. Mag. S. 6. Vol. 1. No. 3. March 1901. 
field due to the moving bodies, must leave the magnetic field unaltered. But this electrification is precisely what would be acquired if the glass tube were (as under ordinary experimental conditions it is) slightly conducting. Such a tube will not destroy the external magnetic field.

11. If we suppose a coil of wire to lie ontside the tube, no lines of force pass through it, and no current will be induced in it by a variation in the velocity of the charged body or of the charge that it carries. Regarding the tube itself as an aggregate of wires parallel to the axis, the same remark applies, and there will be no electromotive force of induction tending to cause a current to flow along it.

12. The equation in the case of a rotating charged disk is different, and cannot be integrated out; but the main difficulty lies in the discussion of the term corresponding to $\tau_{0}$. It seems to be impossible to prove that it is unimportant for our purpose, and it is quite impossible to evaluate it without making assumptions as to the equations satisfied by the vectors of the electromagnetic field at the surface of moving conductors. We cannot say with certainty whether a conducting envelope can, as in the case of $\S 9$, by screening off the electric force reduce the magnetic force also to zero, or whether the surface of the wire in a coil can exert such a screening effect (either partial or total) on the substance of the wire. If a total or considerable partial screening cannot be shown to be theoretically impossible, Crémieu's experiment* does not afford any decisive evidence of the want of truth of the hitherto accepted equations of the electromagnetic field.

XXIX. A Contribution to the Theory of Magnetic Induction in Iron and other Metals. Bu JOHN BUCHANAN, B.Sc.†

TN connexion with his exquisite work relating to the 1 diffusion of heat, Fourier has given us a number of functions with most wonderful properties. To anyone who has made himself acquainted with the graphs which express some of these functions, many of the curves given by Prof. Ewing in his classical researches on magnetic induction must have seemed oddly familiar.

It is well known that magnetic problems can be transformed into problems in electricity and in heat (Clerk Maxwell's Elect. and Mag. 2nd ed. vol. ii. par. 428 et seq.).

The object of this paper, however, is to show-what does

* V. Crémieu, Comptes Rendus, cxxx. p. 1544 (1900)

† Communicated by the Author. 